\title{
On Lars Hörmander's remark on the characteristic Cauchy problem
}

\author{
Jean-Philippe NICOLAS \\ Institut de Mathématiques, M.A.B., Université Bordeaux 1, \\ 351 Cours de la Libération, 33405 Talence Cedex, France. \\ Jean-Philippe.Nicolas@math.u-bordeaux1.fr
}

\begin{abstract}
We extend the results of a work by L. Hörmander 9] concerning the resolution of the characteristic Cauchy problem for second order wave equations with regular first order potentials. The geometrical background of this work was a spatially compact spacetime with smooth metric. The initial data surface was spacelike or null at each point and merely Lipschitz. We lower the regularity hypotheses on the metric and potential and obtain similar results. The Cauchy problem for a spacelike initial data surface is solved for a Lipschitz metric and coefficients of the first order potential that are $L_{\mathrm{loc}}^{\infty}$, with the same finite energy solution space as in the smooth case. We also solve the fully characteristic Cauchy problem with very slightly more regular metric and potential : essentially, a $\mathcal{C}^{1}$ metric and a potential with continuous coefficients of the first order terms and locally $L^{\infty}$ coefficients for the terms of order 0 .
\end{abstract}

\section{Résumé}

Nous étendons des résultats dus à L. Hörmander [9] concernant la résolution du problème de Cauchy caractéristique pour des équations d'onde du second ordre avec un potentiel régulier du premier ordre. Le cadre géométrique de 9 était un espace-temps spatialement compact avec une métrique régulière. L'hypersurface sur laquelle les données initiales sont fixées était spatiale ou caractéristique en chaque point et simplement de régularité Lipschitz. Nous affaiblissons les hypothèses de régularité sur la métrique et le potentiel et nous obtenons des résultats analogues. Le problème de Cauchy pour une hypersurface spatiale est résolu dans le cas d'une métrique Lipschitz et pour un potentiel dont les coefficients sont localement $L^{\infty}$, avec le même espace de solutions que dans le cas régulier. Nous résolvons également le problème de Cauchy totalement caractéristique dans un cadre très légèrement plus régulier : essentiellement, une métrique $\mathcal{C}^{1}$ et un potentiel dont les coefficients des termes du premier ordre sont continus et ceux des termes d'ordre 0 sont localement $L^{\infty}$.

\section{Introduction}

In 1990, in a paper entitled "A remark on the characteristic Cauchy problem" 9], L. Hörmander solved the global Cauchy problem for a class of wave equations on spatially compact space-times with initial data hypersurfaces that were weakly spacelike (i.e. at almost every point either null or spacelike) and merely Lipschitz. He assumed the metric on his space-time to be smooth and the equations he was considering were first order perturbations of the covariant scalar wave equation associated with the metric, the first order perturbation consisting of a differential operator with smooth coefficients. At the end of his work, he remarked that all the estimates depended only on the Lipschitz norm of the metric and the $L^{\infty}$ norms of the coefficients of the first order terms (on a given large enough time interval on which the solution is studied) ; 
he concluded that this was the proper generality of his theorem. However, he did not prove that the result could be extended to the case of a Lipschitz metric and a first order differential perturbation with $L^{\infty}$ coefficients. To this day and to the author's knowledge, this final remark has remained unchecked.

In addition to the mathematical interest of this question, it is relevant for matters related to scattering theory in general relativity in which the author is at present involved : namely, the construction of geometrical versions of scattering theory in generic non-stationary space-times, based on conformal techniques. Such ideas can be traced back to 1963, when R. Penrose put forward in 12 the essential ideas of the resolution of the characteristic Cauchy problem for field equations in relativity. These ideas were then used by F.G. Friedlander to give a conformal construction of scattering theory for the wave equation on static space-times in [7, 8, respectively in 1980 and 2001. In 1990, J.C. Baez, I.E. Segal and Z.F. Zhou applied a similar idea to nonlinear conformally invariant wave equations on flat space-time. On the same year and in the same issue of the same journal, L. Hörmander produced his rigorous proof of the solution to the characteristic Cauchy problem for wave equations on spatially compact space-times [9]. This is fundamental for any conformal description of scattering and Hörmander's proof, based on energy estimates, allows to work with minimum regularity solutions, which is the natural framework of scattering theory. In 2004, L.J. Mason and the author [11] proposed a conformal construction of a scattering operator for spin $1 / 2$ and spin 1 massless fields on generic non stationary asymptotically simple space-times with smooth conformal infinity. This construction uses Penrose's ideas and a modification of Hörmander's proof that allows to establish the equivalence with a standard analytic time-dependent scattering theory, defined in terms of classical wave operators.

The notion of asymptotic simplicity, on which [1] was strongly relating, was defined by R. Penrose, as a simple geometric description of asymptotic flatness. For some time, this was considered by many as an idealised model, because no known solution of the Einstein vacuum equations, except Minkowski space-time, was asymptotically simple. The first examples of vacuum space-times that approached the asymptotically simple framework were the space-times of D. Christodoulou and S. Klainerman 2, propagated from initial data close to Minkowski space. These space-times are non-stationnary and only fail to be asymptotically simple because of a small lack or regularity at null infinity. Since this work, other generically non-stationary vacuum space-times have been constructed, by J. Corvino [5], P. Chrusciel and E. Delay [3, 4] and J. Corvino and R.M. Schoen [6]. They are asymptotically simple with specifyable regularity at null and timelike infinity, and diffeomorphic to Schwarzschild or Kerr spacetime in a neighbourhood of spacelike infinity. Then, S. Klainerman and F. Nicolò [10] proved that for initial data that are close to flat space-time and with stronger fall-off assumptions than in [2], the corresponding global solution of the Einstein vacuum equations is asymptotically simple.

The regularity of conformal infinity encodes the fall-off of the physical metric ; the more regular conformal infinity, the stronger the fall-off. So, working with less regularity of the conformal metric at conformal infinity, means working with larger classes of physical metrics. If in particular one wishes to extend the constructions of [11] to the space-times of [2], then the characteristic Cauchy problem must be solved in frameworks that are only slightly more regular than what L. Hörmander had in mind in his final remark in [9]. It is therefore quite crucial to prove this remark. The present work is a step in this direction. The results of [9] are extended to the regularity setting proposed by Hörmander for the standard Cauchy problem ; we also treat the fully characteristic Cauchy problem (Goursat problem), for this we impose a little more regularity, but we remain below the actual regularity of conformal infinity for the space-times of [2]. The paper is organized as follows.

- In section 2, we describe the geometrical setting and give a summary of L. Hörmander's 
result (theorem 1). His work deals with the Cauchy problem for initial data surfaces that are allowed locally to be spacelike or null and thus solves the standard Cauchy problem as well as the characteristic Cauchy problem.

- Section 3 contains the main results of this work. In subsection 3.1 theorem 2 solves the Cauchy problem in the regularity setting proposed by L. Hörmander. The surprising thing is that the minimum regularity solutions in fact remain continuous in time with values in $H^{1}$ instead of being only locally $L^{\infty}$ with values in $H^{1}$. A corollary for equations that are homogeneous of the second order is then obtained. In this case, we have access to $H^{2}$ solutions for more regular data. Subsection 3.2 treats the fully characteristic Cauchy problem. It uses crucially the corollary of section 3.1 to define a regularization of solutions that has the strong convergence properties appropriate for obtaining the fundamental energy estimates. In theorem 3 the well posedness is proved for a $\mathcal{C}^{1}$ metric and coefficients of lower order terms that are assumed to be continuous for the first order terms and $L_{\mathrm{loc}}^{\infty}$ for the zero-order terms. The proof follows the essential structure of Hörmander's proof, based on two reciprocal energy estimates between some spacelike slice and the null hypersurface, followed by the construction of one solution to the characteristic Cauchy problem. It turns out that the theorem is in fact valid for a regularity setting intermediate between what Hörmander proposed and that of theorem 3 ; this is expressed in theorem 4.

- The proofs of the theorems are given in the last section.

For simplicity, we work with a scalar wave equation with real-valued unknown function. However, the theorems are also valid for a wave equation with complex, tensor or spinor valued unknown function (for spinor fields, provided the space-time admits a spin-structure).

\section{Geometrical and functional framework and summary of Lars Hörmander's result}

The geometrical framework chosen by Hörmander is as follows : $X$ is a $\mathcal{C}^{\infty}$ compact manifold of dimension $n \geq 1$ and $\tilde{X}=\mathbb{R}_{t} \times X$. For $t \in \mathbb{R}$, we denote $X_{t}=\{t\} \times X$. We consider on $X$ a time dependent Riemannian metric $g(t)$ assumed to be $\mathcal{C}^{\infty}$ on $\tilde{X}$. An immediate consequence of this is

Property 1. There exist two continuous positive functions ${ }^{1} C_{1}$ and $C_{2}$ on $\mathbb{R}$ such that, for a given local smooth coordinate system on $X$, the matrix $G=\left(g_{\alpha \beta}\right)$ satisfies, as a quadratic form on $\mathbb{R}^{n}$ :

$$
C_{1}(t) \operatorname{Id}_{n} \leq G(t, x) \leq C_{2}(t) \operatorname{Id}_{n}, \quad \forall(t, x) \in \tilde{X},
$$

where $\operatorname{Id}_{n}$ denotes the $n \times n$ identity matrix.

We also define $\mathrm{d} \nu$ a fixed smooth density on $X$; in local coordinates $\mathrm{d} \nu=\gamma \mathrm{d} x$. We can assume that $\mathrm{d} \nu$ is the volume measure induced by a smooth Riemannian metric $h$ on $X ; \mathrm{d} t \mathrm{~d} \nu$ is then the measure induced by the smooth Riemannian metric $\tilde{h}=\mathrm{d} t^{2}+h$ on $\tilde{X}$. We denote $\nabla$ and $\tilde{\nabla}$ the Levi-Civita connections induced respectively by $h$ and $\tilde{h}$.

We work with Sobolev spaces $H^{\mu}$ and $H_{\mathrm{loc}}^{\mu}$ defined on $X$ and $\tilde{X}$ for any $\mu \in \mathbb{R}$ by local identification with the corresponding function spaces on smooth open sets of $\mathbb{R}^{n}$ and $\mathbb{R}^{n+1}$. We

\footnotetext{
${ }^{1}$ In [9], for simplicity, it is assumed that $C_{1}$ and $C_{2}$ are constants. This is of course unimportant since one can always restrict the study to a generic compact time interval $[-T, T]$.
} 
only use explicit norms for $\mu=0$ or 1 ; in fact we have natural norms on $H^{k}(X)$ and $H^{k}(\tilde{X})$ for any $k \in \mathbb{N}$ :

$$
\begin{gathered}
\|u\|_{H^{k}(X)}^{2}=\sum_{p=0}^{k} \int_{X}\left\langle\nabla^{p} u, \nabla^{p} u\right\rangle \mathrm{d} \nu, \\
\|u\|_{H^{k}(\tilde{X})}^{2}=\sum_{p=0}^{k} \int_{\tilde{X}}\left\langle\tilde{\nabla}^{p} u, \tilde{\nabla}^{p} u\right\rangle \mathrm{d} t \mathrm{~d} \nu
\end{gathered}
$$

where the same notation $\langle.,$.$\rangle refers to the inner product on tensors at a point induced by$ $h$ or $\tilde{h}$. The inner products associated to the norms (11) and (2) are denoted $<.,.\rangle_{H^{k}(X)}$ and $<.,>_{H^{k}(\tilde{X})}$. On $H^{1}(X)$, we also define a norm $\|\cdot\|_{H^{1}\left(X_{t}\right)}$ that is more closely related to the metric $g(t)$ :

$$
\|u\|_{H^{1}\left(X_{t}\right)}^{2}=\int_{X}\left(g^{\alpha \beta}(t, x) \partial_{\alpha} u(x) \partial_{\beta} u(x)+|u(x)|^{2}\right) \mathrm{d} \nu(x) .
$$

The $H^{1}$ norms (1) and (3) are equivalent for any $t \in \mathbb{R}$ and the equivalence is locally uniform in $t$. Another type of function space we shall need to consider is $W^{1, \infty}(\mathcal{O})\left(\right.$ resp. $\left.W_{\text {loc }}^{1, \infty}(\mathcal{O})\right)$, where $\mathcal{O}$ is an open set of $X$ or $\tilde{X}$; it is defined as the space of functions in $L^{\infty}(\mathcal{O})\left(\operatorname{resp} . L_{\text {loc }}^{\infty}(\mathcal{O})\right)$ such that their gradient is also in $L^{\infty}(\mathcal{O})$ (resp. $\left.L_{\text {loc }}^{\infty}(\mathcal{O})\right)$.

On $\tilde{X}$, we consider a wave equation of the form

$$
\square u+L_{1} u=0
$$

where $\square$ denotes the simplified d'Alembertian

$$
\square=\frac{\partial^{2}}{\partial t^{2}}-\gamma^{-1} \frac{\partial}{\partial x^{\alpha}}\left(\gamma g^{\alpha \beta} \frac{\partial}{\partial x^{\beta}}\right),
$$

and $L_{1}$ is a general first order differential operator

$$
L_{1}=b^{0} \frac{\partial}{\partial t}+b^{\alpha} \frac{\partial}{\partial x^{\alpha}}+c
$$

whose coefficients $b^{0}, b^{\alpha}$ and $c$ are assumed to be $\mathcal{C}^{\infty}$ functions on $\tilde{X}$. The hypersurface on which the initial data are specified can be a spacelike Cauchy hypersurface for a standard Cauchy problem, a light cone for a characteristic Cauchy problem (Goursat problem), or anything in between. It is defined as follows

$$
\Sigma=\{(\varphi(x), x) ; x \in X\}, \varphi: X \longrightarrow \mathbb{R},
$$

where $\varphi$ is simply assumed to be Lipschitz on $X$, to allow for singularities such as the vertex of a light cone, and weakly spacelike, i.e.

$$
g^{\alpha \beta}(\varphi(x), x) \partial_{\alpha} \varphi(x) \partial_{\beta} \varphi(x) \leq 1 \text { almost everywhere on } X \text {. }
$$

Condition (8) has a meaning, since Lipschitz functions are differentiable almost everywhere, and it simply says that $\Sigma$ is allowed to be locally spacelike or null but not timelike.

We consider on $\Sigma$ the density measure $\mathrm{d} \nu_{\Sigma}$ which is simply $\mathrm{d} \nu$ lifted to $\Sigma$ using parametrization (7). The hypersurface $\Sigma$ being merely Lipschitz, we can define the spaces $H^{\mu}(\Sigma)$ only for $|\mu| \leq 1$; these spaces are canonically isomorphic to the corresponding Sobolev spaces on $X$ by (77). On $L^{2}(\Sigma)$ and $H^{1}(\Sigma)$, we consider the norms $\|\cdot\|_{L^{2}(\Sigma)}$ and $\|\cdot\|_{H^{1}(\Sigma)}$, naturally induced by 
this isomorphism. We also define a norm $\|\cdot\|_{H^{1}(\Sigma ; g)}$ on $H^{1}(\Sigma)$ in the two following equivalent manners : if the element $\psi$ of $H^{1}(\Sigma)$ is considered as the lift on $\Sigma$ of an element of $H^{1}(X)$, the norm has the form

$$
\|\psi\|_{H^{1}(\Sigma ; g)}^{2}=\int_{X}\left\{|\psi|^{2}+g^{\alpha \beta}(\varphi(x), x) \partial_{\alpha} \psi(x) \partial_{\beta} \psi(x)\right\} \mathrm{d} \nu
$$

and if $\psi$ is defined as the trace on $\Sigma$ of some $\Psi \in H_{\text {loc }}^{3 / 2}(\tilde{X})$,

$$
\|\psi\|_{H^{1}(\Sigma ; g)}^{2}=\int_{\Sigma}\left\{|\Psi|^{2}+g^{\alpha \beta}\left(\partial_{\alpha} \Psi+\partial_{\alpha} \varphi \partial_{t} \Psi\right)\left(\partial_{\beta} \Psi+\partial_{\beta} \varphi \partial_{t} \Psi\right)\right\} \mathrm{d} \nu_{\Sigma} .
$$

The norms $\|\cdot\|_{H^{1}(\Sigma ; g)}$ and $\|\cdot\|_{H^{1}(\Sigma)}$ are of course equivalent. We shall also consider the foliation $\left\{\Sigma_{t}\right\}_{t \in \mathbb{R}}$

$$
\Sigma_{t}=\{(t+\varphi(x), x) ; x \in X\}, \Sigma_{0}=\Sigma .
$$

On each $\Sigma_{t}$, we define the spaces $H^{\mu}\left(\Sigma_{t}\right),-1 \leq \mu \leq 1$. These spaces are canonically isomorphic to the corresponding spaces on $\Sigma$ by parametrizations (17) and (11). We use this canonical isomorphism to identify $H^{\mu}\left(\Sigma_{t}\right)$ with $H^{\mu}(\Sigma)$. On $H^{1}\left(\Sigma_{t}\right)$, in addition to the norm $\|\cdot\|_{H^{1}(\Sigma)}$ inherited from the previous identification, we can also consider a norm involving the restriction to $\Sigma_{t}$ of the metric $g$. Its definition is analogous to (9) and (10): for $\psi \in H^{1}\left(\Sigma_{t}\right)$ seen as the lift on $\Sigma_{t}$ of an element of $H^{1}(X)$,

$$
\|\psi\|_{H^{1}\left(\Sigma_{t} ; g\right)}^{2}=\int_{X}\left\{|\psi|^{2}+g^{\alpha \beta}(t+\varphi(x), x) \partial_{\alpha} \psi(x) \partial_{\beta} \psi(x)\right\} \mathrm{d} \nu
$$

and for $\psi$ defined as the trace on $\Sigma_{t}$ of some $\Psi \in H_{\mathrm{loc}}^{3 / 2}(\tilde{X})$,

$$
\|\psi\|_{H^{1}\left(\Sigma_{t} ; g\right)}^{2}=\int_{\Sigma_{t}}\left\{|\Psi|^{2}+g^{\alpha \beta}\left(\partial_{\alpha} \Psi+\partial_{\alpha} \varphi \partial_{t} \Psi\right)\left(\partial_{\beta} \Psi+\partial_{\beta} \varphi \partial_{t} \Psi\right)\right\} \mathrm{d} \nu_{\Sigma} .
$$

These norms are equivalent, locally uniformly in time, with the $H^{1}(\Sigma)$ norm.

The well-posedness of the Cauchy problem for (4) in $H^{1}(X) \oplus L^{2}(X)$ is well-known : for any initial data $\left(u_{0}, u_{1}\right) \in H^{1}(X) \oplus L^{2}(X)$, for any initial time $s \in \mathbb{R}$, (4) admits a unique solution $u$ in

$$
\mathcal{F}=\mathcal{C}^{0}\left(\mathbb{R}_{t} ; H^{1}(X)\right) \cap \mathcal{C}^{1}\left(\mathbb{R}_{t} ; L^{2}(X)\right)
$$

such that $u(s)=u_{0}$ and $\partial_{t} u(s)=u_{1}$. For $u \in \mathcal{F}$, we introduce the energy of $u$ at time $t$ as the norm of $\left(u(t), \partial_{t} u(t)\right)$ in $H^{1} \oplus L^{2}$ :

$$
E(t, u)=\|u(t)\|_{H^{1}\left(X_{t}\right)}^{2}+\left\|\partial_{t} u(t)\right\|_{L^{2}(X)}^{2}=\int_{X_{t}}\left\{\left|\partial_{t} u\right|^{2}+g^{\alpha \beta} \partial_{\alpha} u \partial_{\beta} \bar{u}+|u|^{2}\right\} \mathrm{d} \nu .
$$

If $u \in \mathcal{F}$ is a solution of (4), it satisfies for all $T>0$ the energy estimate

$$
E(t, u) \leq E(s, u) e^{K_{1}\left(T, g, L_{1}\right)|t-s|} \forall t, s \in[-T, T]
$$

where $K_{1}$ is a continuous positive function of $T>0$, the norms in $W^{1, \infty}(]-T, T[\times X)$ of $g$ and $g^{-1}$ and the norms of the coefficients of $L_{1}$ in $L^{\infty}(]-T, T[\times X)$. We denote by $\mathcal{E}$ the space of finite energy solutions of (4), i.e. the set of solutions of (4) in $\mathcal{F}$. The energy estimate (16) shows that for any $t \in \mathbb{R}$ and for any $T>0$, the following are equivalent norms on $\mathcal{E}$ :

$$
N(t): u \in \mathcal{E} \longmapsto \sqrt{E(t, u)}
$$


and

$$
\|u\|_{\mathcal{F}, T}:=\sup _{-T<\tau<T} N(\tau)(u)
$$

The main result of $[9]$ is the following :

Theorem 1. (Hörmander, 1990) We define on $\Sigma$ the density measure

$$
\mathrm{d} \nu_{\Sigma}^{0}=\left(1-g^{\alpha \beta} \partial_{\alpha} \varphi \partial_{\beta} \varphi\right) \mathrm{d} \nu_{\Sigma},
$$

which is positive where $\Sigma$ is spacelike and vanishes where $\Sigma$ is null, and the associated $L^{2}$ space $L^{2}\left(\Sigma ; \mathrm{d} \nu_{\Sigma}^{0}\right)$. The application

$$
\begin{array}{cccc}
\mathbb{T}_{\Sigma}: & \mathcal{E} & \longrightarrow & H^{1}(\Sigma) \oplus L^{2}\left(\Sigma ; \mathrm{d} \nu_{\Sigma}^{0}\right) \\
u & \longmapsto & \left(u_{\left.\right|_{\Sigma}}, \partial_{t} u_{\left.\right|_{\Sigma}}\right),
\end{array}
$$

which is well defined for smooth solutions, extends as an isomorphism. In particular, there exist $K_{2}\left(T, g, L_{1}\right)$ and $K_{3}\left(T, g, L_{1}\right)$, two positive continuous functions of $T>0$, the norms in $W^{1, \infty}(]-T, T[\times X)$ of $g$ and $g^{-1}$ and the norms of the coefficients of $L_{1}$ in $L^{\infty}(]-T, T[\times X)$, such that for $u \in \mathcal{E}$, for $T>0$ satisfying $-T<\min \{\varphi(x), x \in X\}, T>\max \{\varphi(x), x \in X\}$, we have

$$
\left\|\mathbb{T}_{\Sigma} u\right\|_{1, \Sigma} \leq K_{2}\left(T, g, L_{1}\right)\|u\|_{\mathcal{F}, T}
$$

and

$$
\|u\|_{\mathcal{F}, T} \leq K_{3}\left(T, g, L_{1}\right)\left\|\mathbb{T}_{\Sigma} u\right\|_{1, \Sigma}
$$

where we define

$$
\left\|\mathbb{T}_{\Sigma} u\right\|_{1, \Sigma}^{2}:=\left\|u_{\left.\right|_{\Sigma}}\right\|_{H^{1}(\Sigma ; g)}^{2}+\left\|\partial_{t} u_{\left.\right|_{\Sigma}}\right\|_{L^{2}\left(\Sigma ; \mathrm{d} \nu_{\Sigma}^{0}\right)}^{2} .
$$

Lars Hörmander's proof can be extended with minor modifications to the case where $g$ is in $\mathcal{C}^{2}(\tilde{X})$ and the coefficients of $L_{1}$ are in $W_{\text {loc }}^{1, \infty}(\tilde{X})$ : this guarantees the existence of "regular" solutions living in $H_{\text {loc }}^{2}(\tilde{X})$ which is enough for proving the energy estimates ; the whole proof can then be reproduced using such solutions, instead of the $\mathcal{C}^{\infty}$ solutions used in the smooth case, to approach finite energy solutions. This however is not quite enough for meeting the standards imposed by Hörmander in his final remark.

\section{Main results}

\subsection{The Cauchy problem}

We work on the same geometrical background but we now merely assume the following :

(H1) the metric $g$ is in $\mathcal{C}^{0}(\tilde{X}) \cap W_{\text {loc }}^{1, \infty}(\tilde{X})$ and satisfies Property 1, the coefficients of $L_{1}$ are in $L_{\mathrm{loc}}^{\infty}(\tilde{X})$.

Remark 3.1. The regularity of $\mathrm{d} \nu$ does not need to be lowered since the two operators $\square$ corresponding to two choices of $\mathrm{d} \nu: \mathrm{d} \nu_{1}=\gamma_{1} \mathrm{~d} x, \mathrm{~d} \nu_{2}=\gamma_{2} \mathrm{~d} x$, such that $\gamma_{2}-\gamma_{1} \in W^{1, \infty}(X)$, differ only by a first order operator with bounded coefficients. Hence, the difference between two choices of density is hidden in a black box : the operator $L_{1}$. In fact, if we study the natural covariant wave equation on $\tilde{X}$, this black box already hides the difference between the simplified d'Alembertian $\square$ defined in (5) and the covariant d'Alembertian associated with the Lorentzian metric $\mathrm{d} t^{2}-g$ :

$$
\partial_{t}^{2}-\Delta_{g}=\partial_{t}^{2}-\frac{1}{|\operatorname{det} g|^{\frac{1}{2}}} \partial_{\alpha}\left(|\operatorname{det} g|^{\frac{1}{2}} g^{\alpha \beta} \partial_{\beta}\right)
$$


Because of the lack of regularity of the coefficients of the equation, it is more natural to abandon part of the continuity in time of the solutions. We give a first existence and uniqueness result for solutions that are simply $L_{\mathrm{loc}}^{\infty}$ in time with values in $H^{1}(X)$. Strikingly enough, it is then very easy to show that such solutions are in fact continuous with values in $H^{1}(X)$.

Theorem 2. We introduce the space

$$
\tilde{\mathcal{F}}=L_{\text {loc }}^{\infty}\left(\mathbb{R}_{t} ; H^{1}(X)\right) \cap \mathcal{C}^{1}\left(\mathbb{R}_{t} ; L^{2}(X)\right) .
$$

Under the hypothesis (H1), for any $\left(u_{0}, u_{1}\right) \in H^{1}(X) \oplus L^{2}(X)$, for any $s \in \mathbb{R}$, equation (4) has a unique solution $u \in \tilde{\mathcal{F}}$ such that

$$
u_{\mid t=s}=u_{0}, \partial_{t} u_{\left.\right|_{t=s}}=u_{1} .
$$

Moreover any solution of (4) in $\tilde{\mathcal{F}}$ belongs to $\mathcal{F}$. Therefore, we still denote by $\mathcal{E}$ the space of solutions of (4) in $\tilde{\mathcal{F}}$. The elements of $\mathcal{E}$ satisfy energy estimate (16).

The next result states that when the operator $L_{1}$ is homogeneous of the first order and exactly cancels the first order terms of the d'Alembertian, we can get more regular solutions. This will in particular be crucial for the Goursat problem.

Corollary 3.1. For a metric $g$ in $\mathcal{C}^{0}(\tilde{X}) \cap W_{\text {loc }}^{1, \infty}(\tilde{X})$ that satisfies Property 1 , we consider the equation

$$
\partial_{t}^{2} u-g^{\alpha \beta} \partial_{\alpha} \partial_{\beta} u=0
$$

corresponding to (4) with

$$
L_{1}=\gamma^{-1} \partial_{\alpha}\left(\gamma g^{\alpha \beta}\right) \partial_{\beta} .
$$

Note that $g$ and $L_{1}$ then satisfy hypothesis $(\mathbf{H 1})$. As a consequence of theorem 2 , for any $\left(u_{0}, u_{1}\right) \in H^{1}(X) \oplus L^{2}(X)$, (22) admits in $\tilde{\mathcal{F}}$ a unique solution $u$ such that

$$
u_{\mid t=s}=u_{0}, \partial_{t} u_{\left.\right|_{t=s}}=u_{1}
$$

and we have in fact $u \in \mathcal{F}$. Moreover, if $\left(u_{0}, u_{1}\right) \in H^{2}(X) \oplus H^{1}(X)$, then the solution $u$ satisfies

$$
u \in \bigcap_{l=0}^{2} \mathcal{C}^{l}\left(\mathbb{R}_{t} ; H^{2-l}(X)\right) \text {. }
$$

\subsection{The Goursat problem}

We give an extension of theorem 1 for a metric $g$ that is merely continuously differentiable on $\tilde{X}$ in the case where the hypersurface $\Sigma$ is fully characteristic. More precisely, we assume :

(H2) the metric $g$ is in $\mathcal{C}^{1}(\tilde{X})$, the coefficients of the first order terms of $L_{1}$ are continuous on $\tilde{X}$ and the coefficients of the zero-order terms of $L_{1}$ are in $L_{\mathrm{loc}}^{\infty}(\tilde{X})$.

The hypersurface $\Sigma$ is still defined by (7) where $\varphi: X \rightarrow \mathbb{R}$ is a Lipschitz function, but it is now required to be fully null, that is

$$
g^{\alpha \beta}(x, \varphi(x)) \partial_{\alpha} \varphi(x) \partial_{\beta} \varphi(x)=1 \quad \text { almost everywhere on } X .
$$

Contrary to what one may think, this actually makes things slightly easier since the measure $\mathrm{d} \nu_{\Sigma}^{0}$ vanishes everywhere on $\Sigma$ and therefore the trace of $\partial_{t} u$ on $\Sigma$ is no longer relevant, only the more easily controlled trace of $u$ plays a part in the characteristic Cauchy problem. This is what allows us to extend the results of theorem 1 to the case of a $\mathcal{C}^{1}$ metric. We have the following theorem : 
Theorem 3. Under the assumptions (H1), (H2) and [23) the application

$$
\begin{aligned}
\mathbb{T}_{\Sigma}: \mathcal{E} & \longrightarrow H^{1}(\Sigma) \\
u & \longmapsto u_{\Sigma_{\Sigma}}
\end{aligned}
$$

is well defined and is an isomorphism.

The theorem is actually valid for slightly less regular metric and coefficients of the first order terms. This becomes clear towards the end of the proof, in the only part where we really need more than the minimum regularity setting proposed by Hörmander. The arguments are detailed in remark 4.2

Theorem 4. The result of theorem 3 is still valid under the assumptions (H1), 23) and

$(\widehat{\mathbf{H 2}})$ the metric $g$ is in $L_{\mathrm{loc}}^{\infty}\left(\mathbb{R}_{t} ; \mathcal{C}^{1}(X)\right) \cap W_{\mathrm{loc}}^{1, \infty}\left(\mathbb{R}_{t} ; \mathcal{C}^{0}(X)\right)$, the coefficients of the first order terms of $L_{1}$ are in $L_{\mathrm{loc}}^{\infty}\left(\mathbb{R}_{t} ; \mathcal{C}^{0}(X)\right)$ and the coefficients of the zero-order terms of $L_{1}$ are in $L_{\mathrm{loc}}^{\infty}(\tilde{X})$.

The following additional result is implicit in the proof of theorem 3 :

Corollary 3.2. Let $u \in \mathcal{E}$, we define $v$ as

$$
\begin{aligned}
v: \mathbb{R} & \longrightarrow H^{1}(\Sigma), \\
t & \longmapsto u_{\Sigma_{\Sigma_{t}}} .
\end{aligned}
$$

Then $v \in \mathcal{C}\left(\mathbb{R}_{t} ; H^{1}(\Sigma)\right)$. This can be expressed using the parametrization (11) as follows :

$$
v(t, x):=u(t+\varphi(x), x), v \in\left(\mathbb{R}_{t} ; H^{1}(X)\right) .
$$

\section{Proofs of the main results}

\subsection{Proof of theorem 2}

We shall use the following notations : for $\left.-\infty<t_{1}<t_{2}<+\infty, \mathcal{U}_{t_{1}, t_{2}}:=\right] t_{1}, t_{2}[\times X$ and for $T>0, \Omega_{T}:=\mathcal{U}_{-T, T}$.

- First step : uniqueness. We show that the energy estimate (16) is valid for solutions of (4) in $\tilde{\mathcal{F}}$. We start by establishing an energy estimate for all smooth functions, not assumed to satisfy (4), on $\tilde{X}$. For $v \in \mathcal{C}^{\infty}(\tilde{X})$, if we multiply $\square v+L_{1} v$ by $\partial_{t} v$ and integrate on $\Omega_{T}$ for $T>0$ given, the regularity of $v$ allows us to integrate by parts on $\Omega_{T}$ and to obtain

$$
E(t, v) \leq E(s, v)+2 \int_{\Omega_{T}}\left|\partial_{t} v\right|\left|\square v+L_{1} v\right| \mathrm{d} t \mathrm{~d} \nu+K_{1}\left(T, g, L_{1}\right) \int_{] s, t[} E(\tau, v) \mathrm{d} \tau
$$

where $K_{1}\left(T, g, L_{1}\right)$ is the continuous positive function of $T>0$, the norms in $W^{1, \infty}\left(\Omega_{T}\right)$ of $g$ and $g^{-1}$ and the norms of the coefficients of $L_{1}$ in $L^{\infty}\left(\Omega_{T}\right)$, appearing in (16). By density, estimate (24) carries over to functions $v$ in $H_{\text {loc }}^{2}(\tilde{X})$, but it is not obvious that it remains valid on $\tilde{\mathcal{F}}$ because, using a naive approximation, we cannot make sense either of the convergence of the energy at times $s$ and $t$ or of the convergence of the term containing the d'Alembertian. However, we show that any $u \in \mathcal{E}$ can be approached by a sequence $\left\{u_{k}\right\}_{k}$ of more regular functions such that estimate (24) for $u_{k}$ gives, as $k \rightarrow+\infty$, estimate (16) for $u$. There are three constraints in the construction of the sequence $\left\{u_{k}\right\}_{k}$ : 
1. we must have $E\left(t, u_{k}\right) \rightarrow E(t, u)$ at least for almost every $t$;

2. each $u_{k}$ has to belong to $H_{\text {loc }}^{2}(\tilde{X})$ so as to satisfy (24) ;

3. $\square u_{k}+L_{1} u_{k}$ must tend to zero at least weakly in $L_{\text {loc }}^{2}(\tilde{X})$ in order to obtain

$$
\int_{\Omega_{T}}\left|\partial_{t} u_{k}\right|\left|\square u_{k}+L_{1} u_{k}\right| \mathrm{d} t \mathrm{~d} \nu \longrightarrow 0, \text { as } k \rightarrow+\infty
$$

The first constraint suggests to regularize $u$ in space only. The two other constraints will then automatically be satisfied as well. In the following proposition, we prove the existence of such a regularization in trivial topology, i.e. on $\mathbb{R}_{t} \times \mathbb{R}^{n}$. Then, we use this result locally on $\tilde{X}$ to construct the sequence $\left\{u_{k}\right\}_{k}$.

Proposition 4.1. We consider on $\mathbb{R}^{n}$ a time dependent metric $h(t)$ such that, for all $t_{1}, t_{2}$, $-\infty<t_{1}<t_{2}<+\infty$

$$
\begin{gathered}
h \in \mathcal{C}^{0}\left(\mathbb{R}_{t} \times \mathbb{R}^{n}\right) \cap W^{1, \infty}(] t_{1}, t_{2}\left[\times \mathbb{R}^{n}\right), \\
\exists A, B, 0<A<B<+\infty ; A \operatorname{Id}_{n} \leq h(t, x) \leq B \operatorname{Id}_{n}, \forall(t, x) \in\left[t_{1}, t_{2}\right] \times \mathbb{R}^{n},
\end{gathered}
$$

where $h$ is identified with its matrix in cartesian coordinates. We introduce the operator

$$
\square_{h}=\partial_{t}^{2}-\partial_{\alpha}\left(h^{\alpha \beta} \partial_{\beta}\right)
$$

Let

$$
w \in L_{\mathrm{loc}}^{\infty}\left(\mathbb{R}_{t} ; H^{1}\left(\mathbb{R}^{n}\right)\right) \cap \mathcal{C}^{1}\left(\mathbb{R}_{t} ; L^{2}\left(\mathbb{R}^{n}\right)\right)
$$

such that

$$
\square_{h} w \in L^{2}(] t_{1}, t_{2}\left[\times \mathbb{R}^{n}\right) \forall t_{1}, t_{2} ;-\infty<t_{1}<t_{2}<+\infty .
$$

If we consider on $\mathbb{R}^{n}$ a regularizing sequence (also called approximate identity) defined in the usual manner

$$
\rho \in \mathcal{C}^{\infty}\left(\mathbb{R}^{n}\right), \operatorname{supp} \rho \subset \bar{B}(0,1), \rho \geq 0, \int_{\mathbb{R}^{n}} \rho(x) \mathrm{d} x=1, \rho_{k}(x):=k^{n} \rho(k x) \forall k \in \mathbb{N}^{*},
$$

then the sequence $\left\{w_{k}\right\}_{k}$ defined by convolution of $w$ with $\rho_{k}$ over $\mathbb{R}^{n}$ :

$$
w_{k}(t, x)=\left(w(t) * \rho_{k}\right)(x),
$$

satisfies

(i) $w_{k}(t) \rightarrow w(t)$ in $H^{1}\left(\mathbb{R}^{n}\right)$ for all $t$ such that $w(t) \in H^{1}\left(\mathbb{R}^{n}\right)$;

(ii) $w_{k} \rightarrow w$ in $L_{\text {loc }}^{p}\left(\mathbb{R}_{t} ; H^{1}\left(\mathbb{R}^{n}\right)\right) \cap \mathcal{C}^{1}\left(\mathbb{R}_{t} ; L^{2}\left(\mathbb{R}^{n}\right)\right)$ for all $1 \leq p<+\infty$;

(iii) the sequence $\left\{\square_{h} w_{k}\right\}_{k}$ is bounded in $L_{\text {loc }}^{2}\left(\mathbb{R} \times \mathbb{R}^{n}\right)$;

(iv) $w_{k} \in H_{\text {loc }}^{2}\left(\mathbb{R} \times \mathbb{R}^{n}\right) \forall k \in \mathbb{N}^{*}$.

\section{Proof of proposition 4.1,}

(i) and (ii) are standard. 
(iii) Since $\square_{h} w \in L_{\text {loc }}^{2}\left(\mathbb{R}^{n+1}\right)$, we have

$$
\left(\square_{h} w\right) * \rho_{k} \rightarrow \square_{h} w \text { in } L_{\mathrm{loc}}^{2}\left(\mathbb{R}^{n+1}\right) \simeq L_{\mathrm{loc}}^{2}\left(\mathbb{R}_{t} ; L_{\mathrm{loc}}^{2}\left(\mathbb{R}^{n}\right)\right) .
$$

Hence, $\left(\square_{h} w\right) * \rho_{k}$ is bounded in this space. We consider

$$
\begin{aligned}
\left(\square_{h} w\right) * \rho_{k}-\square_{h} w_{k}= & \left(\partial_{\alpha} h^{\alpha \beta}\right)\left[\left(\partial_{\beta} w\right) * \rho_{k}\right]+h^{\alpha \beta}\left[\left(\partial_{\beta} w\right) *\left(\partial_{\alpha} \rho_{k}\right)\right] \\
& -\left(h^{\alpha \beta} \partial_{\beta} w\right) *\left(\partial_{\alpha} \rho_{k}\right) .
\end{aligned}
$$

The first term is clearly bounded in $L_{\text {loc }}^{2}\left(\mathbb{R}^{n+1}\right)$ since

$$
\partial_{\alpha} h^{\alpha \beta} \in L^{\infty}(] t_{1}, t_{2}\left[\times \mathbb{R}^{n}\right) \text { for any }-\infty<t_{1}<t_{2}<+\infty, \partial_{\beta} w \in L_{\text {loc }}^{2}\left(\mathbb{R}_{t} ; L^{2}\left(\mathbb{R}^{n}\right)\right)
$$

and therefore

$$
\left(\partial_{\alpha} h^{\alpha \beta}\right)\left[\left(\partial_{\beta} w\right) * \rho_{k}\right] \rightarrow\left(\partial_{\alpha} h^{\alpha \beta}\right) \partial_{\beta} w \text { in } L_{\text {loc }}^{2}\left(\mathbb{R}_{t} ; L^{2}\left(\mathbb{R}^{n}\right)\right) .
$$

Now

$$
\begin{aligned}
& \left\{h^{\alpha \beta}\left[\left(\partial_{\beta} w\right) *\left(\partial_{\alpha} \rho_{k}\right)\right]-\left(h^{\alpha \beta} \partial_{\beta} w\right) *\left(\partial_{\alpha} \rho_{k}\right)\right\}(t, x) \\
& =\int_{\operatorname{supp} \rho_{k}}\left\{\left(h^{\alpha \beta}(t, x)-h^{\alpha \beta}(t, x-y)\right) \partial_{\beta} w(t, x-y) \partial_{\alpha} \rho_{k}(y)\right\} \mathrm{d} y .
\end{aligned}
$$

Denoting this quantity $F_{k}(t, x)$, we can estimate it as follows : for $-\infty<t_{1}<t_{2}<+\infty$, denoting $\Omega=] t_{1}, t_{2}\left[\times \mathbb{R}^{n}\right.$, we have for $(t, x) \in \Omega$

$$
\left|F_{k}(t, x)\right| \leq \frac{1}{k}\left\|h^{-1}\right\|_{W^{1, \infty}(\Omega)} \sum_{\alpha, \beta} \int_{\operatorname{supp} \rho_{k}}\left|\partial_{\beta} w(t, x-y)\right| k^{n+1}\left|\partial_{\alpha} \rho(k y)\right| \mathrm{d} y
$$

and putting $\chi_{\alpha, k}(y)=k^{n} \chi_{\alpha}(k y), \chi_{\alpha}(y)=\left|\partial_{\alpha} \rho(y)\right|$,

$$
\left|F_{k}(t, x)\right| \leq\left\|h^{-1}\right\|_{W^{1, \infty}(\Omega)} \sum_{\alpha, \beta}\left(\left|\partial_{\beta} w(t)\right| * \chi_{\alpha, k}\right)(x) .
$$

Using $\left\|\chi_{\alpha, k}\right\|_{L^{1}\left(\mathbb{R}^{n}\right)}=\left\|\chi_{\alpha}\right\|_{L^{1}\left(\mathbb{R}^{n}\right)}$, we obtain

$$
\left\|F_{k}(t)\right\|_{L^{2}\left(\mathbb{R}^{n}\right)} \leq\left\|h^{-1}\right\|_{W^{1, \infty}(\Omega)} \sum_{\alpha, \beta}\left\|\partial_{\beta} w(t)\right\|_{L^{2}\left(\mathbb{R}^{n}\right)}\left\|\chi_{\alpha}\right\|_{L^{1}\left(\mathbb{R}^{n}\right)}
$$

and therefore

$$
\left\|F_{k}\right\|_{L^{2}(\Omega)} \leq C\left\|h^{-1}\right\|_{W^{1, \infty}(\Omega)}\|w\|_{H^{1}(\Omega)},
$$

where $C$ depends only on $\rho$. This proves (iii).

(iv) We have $w \in \mathcal{C}^{1}\left(\mathbb{R}_{t} ; L^{2}\left(\mathbb{R}^{n}\right)\right)$, hence, for each $k \in \mathbb{N}^{*}, w_{k}$ is in $\mathcal{C}^{1}\left(\mathbb{R}_{t} ; \mathcal{C}^{\infty}\left(\mathbb{R}^{n}\right)\right)$. Besides, we have proved that

$$
\square_{h} w_{k}=\partial_{t}^{2} w_{k}-\partial_{\alpha}\left(h^{\alpha \beta} \partial_{\beta} w_{k}\right) \in L_{\mathrm{loc}}^{2}\left(\mathbb{R}^{n+1}\right) .
$$

Also

$$
w_{k} \in \mathcal{C}^{1}\left(\mathbb{R}_{t} ; \mathcal{C}^{\infty}\left(\mathbb{R}^{n}\right)\right) \text { and } h^{\alpha \beta} \in W^{1, \infty}(] t_{1}, t_{2}\left[\times \mathbb{R}^{n}\right) \forall-\infty<t_{1}<t_{2}<+\infty
$$


entail

$$
\partial_{\alpha}\left(h^{\alpha \beta} \partial_{\beta} w_{k}\right) \in L_{\text {loc }}^{2}\left(\mathbb{R}_{t} ; L_{\text {loc }}^{2}\left(\mathbb{R}^{n}\right)\right)
$$

Therefore

$$
\partial_{t}^{2} w_{k} \in L_{\text {loc }}^{2}\left(\mathbb{R}^{n+1}\right)
$$

which proves (iv) and concludes the proof of proposition 4.1 .

We now proceed to constructing the sequence $\left\{u_{k}\right\}_{k}$. We consider :

- $\left\{\Omega^{i}\right\}_{1 \leq i \leq N}$ a covering of $X$ by open sets of trivial topology ;

- $\left\{\mathcal{U}^{i}\right\}_{1 \leq i \leq N}$ a covering of $X$ by open sets such that $\overline{\mathcal{U}^{i}} \subset \Omega^{i}$;

- $\left\{\chi^{i}\right\}_{1 \leq i \leq N}$ a partition of unity associated with $\left\{\mathcal{U}^{i}\right\}$, i.e.

$$
\chi^{i} \in \mathcal{C}^{\infty}(X), \operatorname{supp} \chi^{i} \subset \overline{\mathcal{U}^{i}}, 0 \leq \chi^{i} \leq 1, \sum_{i=1}^{N} \chi^{i}=1 \text { on } X .
$$

Putting $v^{i}=\chi^{i} u$, we clearly have $v^{i} \in \tilde{\mathcal{F}}$ and

$$
\square v^{i}=\chi^{i} \square u-\gamma^{-1} \partial_{\alpha}\left(\gamma g^{\alpha \beta}\left(\partial_{\beta} \chi^{i}\right) u\right)-g^{\alpha \beta}\left(\partial_{\alpha} \chi^{i}\right)\left(\partial_{\beta} u\right) \in L_{\mathrm{loc}}^{2}(\tilde{X}) .
$$

For $1 \leq i \leq N, \Omega^{i}$ is a trivial topology open subset of $X$; it can therefore be identified, by means of a global coordinate system, with a bounded open set $\Omega^{i}$ in $\mathbb{R}^{n}$. The metric $g_{\left.\right|_{\mathbb{R}_{t} \times \Omega^{i}}}$ can be extended as a function ${ }^{i} h(t, x)$ on $\mathbb{R}_{t} \times \mathbb{R}^{n}$ satisfying (25) and (26). The functions $v^{i}$ and $\square v^{i}$ on $\tilde{X}$ have their support in $\mathbb{R}_{t} \times \overline{\mathcal{U}^{i}} \subset \mathbb{R}_{t} \times \Omega^{i}$ and can therefore be considered as functions on $\mathbb{R}_{t} \times \mathbb{R}^{n}$. Then, $v^{i}$ and

$$
\square_{i h} v^{i}=\square v^{i}+\text { first order terms in } L_{\text {loc }}^{2}\left(\mathbb{R}_{t} ; L^{2}\left(\Omega^{i}\right)\right)
$$

satisfy the hypotheses of proposition 4.1. Hence, for each $1 \leq i \leq N$, we can construct the sequence

$$
v_{k}^{i}=v^{i} * \rho_{k}, k \in \mathbb{N}^{*}
$$

and it will satisfy properties (i)-(iv). In addition,

$$
\operatorname{supp} v_{k}^{i} \subset \mathbb{R}_{t} \times\left(\overline{\mathcal{U}^{i}}+\bar{B}\left(0, \frac{1}{k}\right)\right) \text { in } \mathbb{R} \times \mathbb{R}^{n}
$$

whence for $k$ large enough, $\square_{i h} v_{k}^{i}$ and $v_{k}^{i}$ have their support in $\mathbb{R}_{t} \times \Omega^{i}$ and can be considered as functions on $\tilde{X}$. In this manner, for a given $\bar{k} \in \mathbb{N}^{*}$, we obtain a sequence $\left\{u_{k}\right\}_{k \geq \bar{k}}$ defined by

$$
u_{k}=\sum_{i=1}^{N} v_{k}^{i}
$$

such that

$$
\begin{gathered}
u_{k} \in H_{\mathrm{loc}}^{2}(\tilde{X}), \\
u_{k}(t) \rightarrow u(t) \text { in } H^{1}(X), \forall t \text { such that } u(t) \in H^{1}(X), \\
u_{k} \rightarrow u \text { in } L_{\text {loc }}^{p}\left(\mathbb{R}_{t} ; H^{1}(X)\right) \cup \mathcal{C}^{1}\left(\mathbb{R}_{t} ; L^{2}(X)\right) \forall p, 1 \leq p<+\infty, \\
\square u_{k}+L_{1} u_{k} \text { bounded in } L_{\text {loc }}^{2}(\tilde{X}) .
\end{gathered}
$$


Note that (31) is an easy consequence of property (iii) for $v_{i}^{k}$ since

$$
\square u_{k}+L_{1} u_{k}-\sum_{i=1}^{N} \square_{i h} v_{k}^{i}
$$

is a sum of first or zero order derivatives of the $v_{k}^{i}$ with coefficients in $L_{\text {loc }}^{\infty}(\tilde{X})$ and these terms converge in $L_{\text {loc }}^{2}(\tilde{X})$ by (30). By (28), each $u_{k}$ satisfies (24) : for all $T>0$, for all $t, s \in[-T, T]$,

$$
E\left(t, u_{k}\right) \leq E\left(s, u_{k}\right)+2 \int_{\Omega_{T}}\left|\partial_{t} u_{k}\right|\left|\square u_{k}+L_{1} u_{k}\right| \mathrm{d} t \mathrm{~d} \nu+K_{1}\left(T, g, L_{1}\right) \int_{] s, t[} E\left(\tau, u_{k}\right) \mathrm{d} \tau .
$$

Properties (29) and (30) imply that for almost all $s, t \in[-T, T]$ (more precisely for all $s, t$ such that both $u(s)$ and $u(t)$ belong to $\left.H^{1}(X)\right)$

$$
E\left(t, u_{k}\right) \rightarrow E(t, u) \text { and } E\left(s, u_{k}\right) \rightarrow E(s, u) .
$$

Property (30) also entails the convergence of the last term of the inequality

$$
\int_{] s, t[} E\left(\tau, u_{k}\right) \mathrm{d} \tau \rightarrow \int_{] s, t[} E(\tau, u) \mathrm{d} \tau
$$

Extracting a subsequence if necessary, (31) entails that $\square u_{k}+L_{1} u_{k}$ converges weakly in $L^{2}\left(\Omega_{T}\right)$, the limit being zero since, by (29) and using the fact that $u$ is a solution of (4), $\square u_{k}+L_{1} u_{k}$ converges towards zero in $\mathcal{D}^{\prime}\left(\Omega_{T}\right)$, the space of distributions on $\Omega_{T}$. Besides, $\partial_{t} u_{k}$ converges strongly towards $\partial_{t} u$ in $L^{2}\left(\Omega_{T}\right)$, whence

$$
\int_{\Omega_{T}}\left|\partial_{t} u_{k}\right|\left|\square u_{k}+L_{1} u_{k}\right| \mathrm{d} t \mathrm{~d} \nu \longrightarrow 0
$$

Consequently, for all $t, s \in[-T, T]$ such that $u(t), u(s) \in H^{1}(X)$,

$$
E(t, u) \leq E(s, u)+K_{1}\left(T, g, L_{1}\right) \int_{] s, t[} E(\tau, u) \mathrm{d} \tau
$$

This gives (16) for $u$ in the following sense : if $u(s) \in H^{1}(X), s \in[-T, T]$, then for almost all $t \in[-T, T]$,

$$
E(t, u) \leq E(s, u) e^{K_{1}\left(T, g, L_{1}\right)|t-s|} .
$$

The uniqueness of solutions to the Cauchy problem for (4) in $\tilde{\mathcal{F}}$ follows.

- Second step : existence. Let $(\phi, \psi) \in H^{1}(X) \oplus L^{2}(X), s \in \mathbb{R}$, we wish to find $u \in \tilde{\mathcal{F}}$ such that

$$
\square u+L_{1} u=0, u_{\mid t=s}=\phi, \partial_{t} u_{\mid t=s}=\psi .
$$

In order to use the well posedness of the Cauchy problem in the smooth case, we regularize the metric and the coefficients of $L_{1}$. For $k \in \mathbb{N}^{*}$, we define

(a) ${ }^{k} g$ a time-dependent riemannian metric on $X,{ }^{k} g \in \mathcal{C}^{\infty}(\tilde{X})$;

(b) ${ }^{k} L_{1}={ }^{k} b^{0} \partial_{t}+{ }^{k} b^{\alpha} \partial_{\alpha}+{ }^{k} c,{ }^{k} b^{0},{ }^{k} b^{\alpha},{ }^{k} c \in \mathcal{C}^{\infty}(\tilde{X})$; 
such that :

$$
\begin{aligned}
& { }^{k} g \longrightarrow g \text { in } \mathcal{C}^{0}(\tilde{X}) \cap H_{\text {loc }}^{1}(\tilde{X}) ; \\
& { }^{k} g \text { bounded in } W_{\text {loc }}^{1, \infty}(\tilde{X}) \text {; } \\
& \left.\begin{array}{rlc}
{ }^{k} c & \longrightarrow & c \\
{ }^{k} b^{0} & \longrightarrow & b^{0} \\
{ }^{k} b^{\alpha} & \longrightarrow & b^{\alpha}
\end{array}\right\} \text { in } L_{\mathrm{loc}}^{p}(\tilde{X}) \forall p ; 1 \leq p<+\infty ; \\
& { }^{k} b^{0},{ }^{k} b^{\alpha},{ }^{k} c \text { bounded in } L_{\text {loc }}^{\infty}(\tilde{X})
\end{aligned}
$$

and there exist two positive continuous functions $D_{1}$ and $D_{2}$ such that

$$
\forall k \in \mathbb{N}^{*}, \forall(t, x) \in \tilde{X}, D_{1}(t) \operatorname{Id}_{n} \leq{ }^{k} g_{\alpha \beta}(t, x) \leq D_{2}(t) \operatorname{Id}_{n} .
$$

Typically, such sequences are constructed using coordinate charts and, in each domain, convolution by a regularizing sequence on $\mathbb{R}_{t} \times \mathbb{R}^{n}$; this is similar to what we did for constructing the sequence $u_{k}$ in the first step of the proof, but now, the regularizing sequence and the convolution involve time as well as space variables.

For each $k$, we consider the equation

$$
\frac{\partial^{2} v}{\partial t^{2}}-\gamma^{-1} \frac{\partial}{\partial x^{\alpha}}\left(\gamma\left({ }^{k} g^{\alpha \beta}\right) \frac{\partial v}{\partial x^{\beta}}\right)+{ }^{k} L_{1} v=0 .
$$

Theorem 1 tells us that (40) has a unique solution $v_{k} \in \mathcal{F}$ such that $v_{k}(s)=\phi$ and $\partial_{t} v_{k}(s)=\psi$. This solution satisfies the energy estimate

$$
\forall T>|s|, \forall t \in[-T, T], E_{k}\left(t, v_{k}\right) \leq E_{k}\left(s, v_{k}\right) e^{K_{1}\left(T,{ }^{k},{ }^{k} L_{1}\right)|t-s|},
$$

where $E_{k}$ is the energy (15) defined using the metric ${ }^{k} g$ instead of $g . E_{k}(t,$.$) , just like E(t,$.$) ,$ is (uniformly in $k$ and locally uniformly in time) equivalent to the norm in $H^{1}(X) \oplus L^{2}(X)$. Besides, (36) and (38) imply that $\left\{K_{1}\left(T,{ }^{k} g,{ }^{k} L_{1}\right)\right\}_{k}$ is bounded in $\mathbb{R}^{+}$. The upshot of all this is that $\left\{v_{k}\right\}_{k}$ is bounded in $\mathcal{F}$. Hence, for $T>|s|$ fixed, extracting a subsequence if necessary, we can assume the convergence of $\left\{v_{k}\right\}$ in the following spaces (we call $u$ the common limit) :

$$
\begin{gathered}
v_{k} \rightarrow u \text { in } H^{1}\left(\Omega_{T}\right)-w, \\
v_{k} \rightarrow u \text { in } H^{\mu}\left(\Omega_{T}\right), \forall \mu<1,
\end{gathered}
$$

where " $-w$ " denotes the weak topology. Hence, by standard trace theorems

$$
v_{k} \rightarrow u \text { in } \mathcal{C}\left([-T, T] ; L^{2}(X)\right)
$$

and by the Banach-Alaoglou theorem

$$
\begin{gathered}
v_{k} \rightarrow u \text { in } L^{\infty}(]-T, T\left[; H^{1}(X)\right)-w-*, \\
\partial_{t} v_{k} \rightarrow \partial_{t} u \text { in } L^{\infty}(]-T, T\left[; L^{2}(X)\right)-w-*,
\end{gathered}
$$

where " $-w-*$ " denotes the weak star topology. Now the convergences (42), (35) and (37) imply

$$
\left.\begin{array}{rl}
\partial_{t}^{2} v_{k} & \longrightarrow \partial_{t}^{2} u \\
\gamma^{-1} \partial_{\alpha}\left(\gamma\left({ }^{k} g^{\alpha \beta}\right) \partial_{\beta} v_{k}\right) & \longrightarrow \gamma^{-1} \partial_{\alpha}\left(\gamma g^{\alpha \beta} \partial_{\beta} u\right) \\
{ }^{k} L_{1} v_{k} & \longrightarrow L_{1} u
\end{array}\right\} \text { in } \mathcal{D}^{\prime}\left(\Omega_{T}\right),
$$


whereby $u$ satisfies equation (44) in the sense of distributions on $\Omega_{T}$. Using uniqueness, we have thus constructed a solution $u$ of (41) defined on $\tilde{X}$ and that belongs to $\tilde{\mathcal{F}}$. Indeed, we know that

$$
u \in L_{\mathrm{loc}}^{\infty}\left(\mathbb{R}_{t} ; H^{1}(X)\right), \partial_{t} u \in L_{\mathrm{loc}}^{\infty}\left(\mathbb{R}_{t} ; L^{2}(X)\right) .
$$

Since $g \in W_{\mathrm{loc}}^{1, \infty}(\tilde{X})$ and the coefficients of $L_{1}$ are in $L_{\mathrm{loc}}^{\infty}(\tilde{X})$, (4) entails

$$
\partial_{t}^{2} u=\gamma^{-1} \partial_{\alpha}\left(\gamma g^{\alpha \beta} \partial_{\beta} u\right)-L_{1} u \in L_{\mathrm{loc}}^{\infty}\left(\mathbb{R}_{t} ; H^{-1}(X)\right) .
$$

Using J.-L. Lions's principle of intermediate derivatives, (477) and (48) imply

$$
\partial_{t} u \in \mathcal{C}\left(\mathbb{R}_{t} ; L^{2}(X)\right)
$$

and therefore $u \in \tilde{\mathcal{F}}$. The last things to check are the two initial data conditions. The initial value of $u$ is easy ; using (44)

$$
v_{k}(s)=\phi \longrightarrow u(s) \text { in } L^{2}(X)
$$

whence $u(s)=\phi$. The trace of $\partial_{t} u$ at $t=s$ requires more care. We write

$$
\partial_{t}^{2}\left(u-v_{k}\right)=\gamma^{-1} \partial_{\alpha}\left(\gamma\left[{ }^{k} g^{\alpha \beta} \partial_{\beta} v_{k}-g^{\alpha \beta} \partial_{\beta} u\right]\right)+{ }^{k} L_{1} v_{k}-L_{1} u
$$

(36), (38) and (42) imply the boundedness in $L^{2}(]-T, T\left[; H^{-1}(X)\right)$ of $\partial_{t}^{2}\left(u-v_{k}\right)$. This allows us, first, to write for $t \in[-T, T], t_{0}$ fixed in $[-T, T]$,

$$
\partial_{t} u(t)-\partial_{t} v_{k}(t)=\partial_{t} u\left(t_{0}\right)-\partial_{t} v_{k}\left(t_{0}\right)+\int_{] t_{0}, t[} \partial_{t}^{2}\left(u-v_{k}\right)(\tau) \mathrm{d} \tau
$$

second, extracting another subsequence if necessary, to assume

$$
\partial_{t}^{2}\left(u-v_{k}\right) \longrightarrow 0 \text { in } L^{2}(]-T, T\left[; H^{-1}(X)\right)-w .
$$

This last convergence gives

$$
\int_{] t_{0}, t[} \partial_{t}^{2}\left(u-v_{k}\right)(\tau) \mathrm{d} \tau \longrightarrow 0 \text { in } L^{2}(]-T, T\left[; H^{-1}(X)\right)-w .
$$

Since (42) implies

$$
\partial_{t} v_{k} \rightarrow \partial_{t} u \text { in } L^{2}\left(\Omega_{T}\right)-w \hookrightarrow L^{2}(]-T, T\left[; H^{-1}(X)\right)-w,
$$

we deduce from (49) that

$$
\partial_{t} v_{k}\left(t_{0}\right) \longrightarrow \partial_{t} u\left(t_{0}\right) \text { in } H^{-1}(X)-w, \forall t_{0} \in[-T, T] .
$$

In particular, for $t_{0}=s$,

$$
\Psi=\partial_{t} v_{k}(s) \longrightarrow \partial_{t} u(s) \text { in } H^{-1}(X)-w
$$

which gives us $\partial_{t} u(s)=\Psi$ and concludes the second part of the proof of theorem 2

- Third step : continuity in time of the solutions. We consider $u$ the unique solution in $\tilde{\mathcal{F}}$ of the Cauchy problem (34), $T>|s|$ and $v_{k}$ the sequence constructed in the second step of the proof. For any fixed $t$ in $[-T, T]$, the energy estimate (41) implies that $\left\{v_{k}(t)\right\}_{k}$ is bounded 
in $H^{1}(X)$. Hence, extracting a subsequence if necessary, we can assume that $v_{k}(t)$ converges weakly in $H^{1}(X)$. This together with the strong convergence (44) guarantees that $u(t)$ belongs to $H^{1}(X)$. The construction of the sequence $v_{k}$ can be made for any fixed $T>|s|$. It therefore turns out that

$$
u(t) \in H^{1}(X) \forall t \in \mathbb{R}
$$

and hence, the energy estimate (33) is valid for all $t, s$. This implies in particular that for any solution $u$ of (4) in $\tilde{\mathcal{F}}$, the energy $E(t, u)$ is continuous in time. Besides, it is easy to show that

$$
u \in \tilde{\mathcal{F}} \Longrightarrow u \in \mathcal{C}\left(\mathbb{R}_{t} ; H^{1}(X)-w\right)
$$

The continuity of the energy therefore entails the strong continuity of $u$ in time with values in $H^{1}(X)$, which proves $u \in \mathcal{F}$. We now prove (150). Let $v \in \tilde{\mathcal{F}}$ and $w \in H^{1}(X)$. We put for $t \in \mathbb{R}$

$$
f(t)=\langle v(t), w\rangle_{H^{1}(X)} .
$$

Given $t_{0} \in \mathbb{R}$ we show the continuity of $f$ at $t_{0}$. Let $t_{n} \rightarrow t_{0}$ and $\left\{w_{k}\right\}_{k}$ a sequence in $\mathcal{C}^{\infty}(X)$ converging towards $w$ in $H^{1}(X)$. For each $k$, using $v \in \mathcal{C}\left(\mathbb{R}_{t} ; L^{2}(X)\right)$, we have

$$
\left\langle v\left(t_{n}\right)-v\left(t_{0}\right), w_{k}\right\rangle_{H^{1}(X)}=\left\langle v\left(t_{n}\right)-v\left(t_{0}\right),\left(1-\Delta_{h}\right) w_{k}\right\rangle_{L^{2}(X)} \longrightarrow 0, n \rightarrow+\infty,
$$

where $\Delta_{h}=\gamma^{-1} \partial_{\alpha}\left(\gamma h^{\alpha \beta} \partial_{\beta}\right)$ is the Laplacian associated with the metric $h$ on $X$, introduced in section 2 to define the $H^{1}$ norm on $X$, and to which the measure $\mathrm{d} \nu$ is associated. We write

$$
\left\langle v\left(t_{n}\right)-v\left(t_{0}\right), w\right\rangle_{H^{1}(X)}=\left\langle v\left(t_{n}\right)-v\left(t_{0}\right), w-w_{k}\right\rangle_{H^{1}(X)}+\left\langle v\left(t_{n}\right)-v\left(t_{0}\right), w_{k}\right\rangle_{H^{1}(X)} .
$$

Consider $\varepsilon>0$. Using the fact that $v \in L_{\text {loc }}^{\infty}\left(\mathbb{R}_{t} ; H^{1}(X)\right)$, we choose $k$ large enough so that for all $n$

$$
\left|\left\langle v\left(t_{n}\right)-v\left(t_{0}\right), w-w_{k}\right\rangle_{H^{1}(X)}\right| \leq \varepsilon / 2,
$$

then, for this value of $k$, we choose $n$ large enough so that

$$
\left|\left\langle v\left(t_{n}\right)-v\left(t_{0}\right), w_{k}\right\rangle_{H^{1}(X)}\right| \leq \varepsilon / 2 .
$$

This proves the continuity of $f(t)$ and concludes the proof of theorem 2

\subsection{Proof of corollary 3.1}

First, we write (22) as a special case of (44):

$$
\partial_{t}^{2} u-g^{\alpha \beta} \partial_{\alpha} \partial_{\beta} u=\square u+\gamma^{-1} \partial_{\alpha}\left(\gamma g^{\alpha \beta}\right) \partial_{\beta} u=0 .
$$

Since $g, g^{-1} \in W_{\text {loc }}^{1, \infty}(\tilde{X})$, the coefficients of the first order operator clearly belong to $L_{\text {loc }}^{\infty}(\tilde{X})$ and we are in the framework of theorem 2. To check that we can get more regular solutions, we simply apply a partial derivation to (22) :

$$
\partial_{\mu}\left(\partial_{t}^{2} u-g^{\alpha \beta} \partial_{\alpha} \partial_{\beta} u\right)=\left(\partial_{t}^{2}-g^{\alpha \beta} \partial_{\alpha} \partial_{\beta}\right) \partial_{\mu} u-\left(\partial_{\mu} g^{\alpha \beta}\right) \partial_{\alpha} \partial_{\beta} u .
$$

We can therefore write the following system of equations :

$$
\left\{\begin{aligned}
\left(\partial_{t}^{2}-g^{\alpha \beta} \partial_{\alpha} \partial_{\beta}\right) u & =0 \\
\left(\partial_{t}^{2}-g^{\alpha \beta} \partial_{\alpha} \partial_{\beta}\right) \partial_{\mu} u & =\left(\partial_{\mu} g^{\alpha \beta}\right) \partial_{\alpha} \partial_{\beta} u, \mu=1, \ldots, n
\end{aligned}\right.
$$


This system is of the form

$$
\left(\partial_{t}^{2}-g^{\alpha \beta} \partial_{\alpha} \partial_{\beta}\right) U=L_{1} U, U={ }^{t}\left(u, \partial_{1} u, \ldots, \partial_{n} u\right),
$$

where $L_{1}$ is a first order differential operator whose coefficients belong to $L_{\mathrm{loc}}^{\infty}(\tilde{X})$ since the metric $g$ is in $W_{\text {loc }}^{1, \infty}(\tilde{X})$. By theorem 2 (in the case where the unknown function is a vector field), the system (51) admits a well-posed Cauchy problem in $\tilde{\mathcal{F}}$ and the solutions belong to $\mathcal{F}$. This guarantees the additional regularity of solutions of (22) for data in $H^{2} \oplus H^{1}$ and concludes the proof of corollary 3.1

\subsection{Proof of theorem 3 ,}

- Inequalities (20) and (21). To prove these inequalities with our regularity assumptions, we define a regularization of the solution $u$ by functions $u_{k}$ in $H_{\text {loc }}^{2}(\tilde{X})$ that satisfy estimates of type (20)-(21), with constants uniform in $k$, and that converge towards $u$ strongly in $H^{1}\left(X_{t}\right)$ for all $t$ and in $H^{1}(\Sigma)$. This makes a crucial use of corollary [3.1]

We write equation (4) as follows

$$
\partial_{t}^{2} u-g^{\alpha \beta} \partial_{\alpha} \partial_{\beta} u+\tilde{L}_{1} u=0
$$

where

$$
\begin{gathered}
\tilde{L}_{1}=-\gamma^{-1}\left(\partial_{\alpha}\left(\gamma g^{\alpha \beta}\right)\right) \partial_{\beta}+L_{1}=: p^{0} \partial_{t}+p^{\beta} \partial_{\beta}+q, \\
p^{0}=b^{0}, p^{\beta}=-\gamma^{-1}\left(\partial_{\alpha}\left(\gamma g^{\alpha \beta}\right)\right)+b^{\beta}, q=c .
\end{gathered}
$$

The coefficients of $\tilde{L}_{1}$ satisfy

$$
p^{0}, p^{\beta} \in \mathcal{C}^{0}(\tilde{X}), q \in L_{\mathrm{loc}}^{\infty}(\tilde{X}) .
$$

We define an approximation of equation (52) in which only the coefficients of $\tilde{L}_{1}$ are regularized :

$$
\partial_{t}^{2} u-g^{\alpha \beta} \partial_{\alpha} \partial_{\beta} u+\tilde{L}_{1}^{k} u=0, \tilde{L}_{1}^{k}=p_{k}^{0} \partial_{t}+p_{k}^{\beta} \partial_{\beta}+q_{k},
$$

where the coefficients of $\tilde{L}_{1}^{k}$ satisfy

$$
\begin{gathered}
p_{k}^{0}, p_{k}^{\beta}, q_{k} \in \mathcal{C}^{\infty}(\tilde{X}), \\
p_{k}^{0} \rightarrow p^{0} \text { in } \mathcal{C}(\tilde{X}), p_{k}^{\beta} \rightarrow p^{\beta} \text { in } \mathcal{C}(\tilde{X}), \\
q_{k} \rightarrow q \text { in } L_{\text {loc }}^{p}(\tilde{X}), \forall 1 \leq p<+\infty, \\
q_{k} \text { bounded in } L^{\infty}(]-T, T[\times X) \forall T>0 .
\end{gathered}
$$

Let $\left(u_{0}, u_{1}\right) \in H^{1}(X) \oplus L^{2}(X)$. We consider $u \in \mathcal{F}$ the solution of (52) such that $u(0)=u_{0}$ and $\partial_{t} u(0)=u_{1}$. We also consider some sequences $\left\{u_{0}^{k}\right\}_{k}$ and $\left\{u_{1}^{k}\right\}_{k}$ of smooth functions on $X$ such that

$$
u_{0}^{k} \rightarrow u_{0} \text { in } H^{1}(X) \text { and } u_{1}^{k} \rightarrow u_{1} \text { in } L^{2}(X) .
$$

Let $u_{k} \in H_{\mathrm{loc}}^{2}(\tilde{X})$ the solution in $\mathcal{F}$ of (53) such that $u_{k}(0)=u_{0}^{k}$ and $\partial_{t} u_{k}(0)=u_{1}^{k}$. For each $k, u_{k}$ satisfies estimates of type (20) and (21) uniformly in $k$, more precisely there exists $C>0$ such that, for all $k$ :

$$
\begin{array}{r}
\left\|u_{\left.k\right|_{\Sigma}}\right\|_{1, \Sigma}^{2} \leq C\left(\left\|u_{0}^{k}\right\|_{H^{1}(X)}^{2}+\left\|u_{1}^{k}\right\|_{L^{2}(X)}^{2}\right) \\
\left\|u_{0}^{k}\right\|_{H^{1}(X)}^{2}+\left\|u_{1}^{k}\right\|_{L^{2}(X)}^{2} \leq C\left\|u_{\left.k\right|_{\Sigma}}\right\|_{1, \Sigma}^{2}
\end{array}
$$


In order to establish (20) and (21) for $u$, we only need to prove that $u_{k}$ converges towards $u$ in $H^{1}(\Sigma)$, since

$$
\left\|u_{0}^{k}\right\|_{H^{1}(X)}^{2}+\left\|u_{1}^{k}\right\|_{L^{2}(X)}^{2} \rightarrow\left\|u_{0}\right\|_{H^{1}(X)}^{2}+\left\|u_{1}\right\|_{L^{2}(X)}^{2}=E(0, u) \text { as } k \rightarrow+\infty .
$$

We will use the following proposition.

Proposition 4.2. We consider the equation

$$
\partial_{t}^{2} v-g^{\alpha \beta} \partial_{\alpha \beta}^{2} v+L v=f
$$

where $L$ is a first order differential operator with smooth coefficients on $\tilde{X}$ and the source $f$ belongs to $L_{\text {loc }}^{2}(\tilde{X})$.

1. We consider $T>0$ and $\left.\Omega_{T}=\right]-T, T[\times X$, there exists a continuous positive function $C_{1}(T, g, L)$ of $T$, the norms of $g$ and $g^{-1}$ in $W^{1, \infty}\left(\Omega_{T}\right)$ and the norms of the coefficients of $L$ in $L^{\infty}\left(\Omega_{T}\right)$, such that, for any solution $v$ of (61) in $\mathcal{F}$ and for all $t, s \in[-T, T]$ :

$$
E(t, v) \leq C_{1}(T, g, L)\left(E(s, v)+\|f\|_{L^{1}(]-T, T\left[; L^{2}(X)\right)}^{2}\right) .
$$

2. We now consider $T>\max \{|\min \varphi|,|\max \varphi|\}$. There exist continuous positive functions $C_{2}(T, g, L), C_{3}(T, g, L, f)$ of $T$, the norms of $g$ and $g^{-1}$ in $W^{1, \infty}\left(\Omega_{T}\right)$ and the norms of the coefficients of $L$ in $L^{\infty}\left(\Omega_{T}\right)$, such that, for any solution $v$ of (61) in $H_{\mathrm{loc}}^{2}(\tilde{X})$ and for any $s \in[-T, T]$, we have ${ }^{2}:$

$$
\begin{gathered}
\left\|v_{\left.\right|_{\Sigma}}\right\|_{H^{1}(\Sigma)} \leq C_{2}(T, g, L)\left(E(s, v)+\|f\|_{L^{1}(]-T, T\left[; L^{2}(X)\right)}^{2}\right), \\
E(s, v) \leq C_{3}(T, g, L)\left(\left\|v_{\left.\right|_{\Sigma}}\right\|_{H^{1}(\Sigma)}+\|f\|_{L^{2}\left(\Omega_{T}\right)}^{2}\right) .
\end{gathered}
$$

Remark 4.1. Estimate (64) will not be useful to us, we have given it for completeness.

\section{Proof.}

1. Proof of (62). We have obtained in the proof of theorem 2 that estimate (16) is valid for solutions of (4) in $\mathcal{F}$ under the assumption (H1). If we consider some source $f$ in $L_{\text {loc }}^{1}\left(\mathbb{R}_{t} ; L^{2}(X)\right)$, we still have existence and uniqueness in $\mathcal{F}$ of the solutions of

$$
\square v+L_{1} v=f
$$

and these solutions are given in terms of their initial data at time $s$ by the Duhamel formula ${ }^{3}$

$$
\left(\begin{array}{c}
v(t) \\
\partial_{t} v(t)
\end{array}\right)=\mathcal{U}(t, s)\left(\begin{array}{c}
v(s) \\
\partial_{t} v(s)
\end{array}\right)+\int_{s}^{t} \mathcal{U}(t, \tau)\left(\begin{array}{c}
0 \\
f(\tau)
\end{array}\right) \mathrm{d} \tau
$$

where $\mathcal{U}(t, s)$ denotes the propagator for equation (4), that to initial data ${ }^{t}\left(u(s), \partial_{t} u(s)\right)$, associates the solution at time $t:{ }^{t}\left(u(t), \partial_{t} u(t)\right)$. Equation (61) in the source-free case can be written as

$$
\square v+\gamma^{-1}\left(\partial_{\alpha}\left(\gamma g^{\alpha \beta}\right)\right) \partial_{\beta} v+L v=0
$$

\footnotetext{
${ }^{2}$ The existence of such solutions is not guaranteed in the general case because of the low regularity of $f$, but we will use this proposition in cases where we know such solutions, namely the functions $u_{k}$ or rather the difference $u_{k}-u_{l}$ between two such solutions ; see equation (67).

${ }^{3}$ This is established by a standard fixed point argument.
} 
and therefore the solutions satisfy estimate (16) with a bound $e^{K_{1}(T, g, L)|t-s|}$ where $K_{1}$ is a continuous positive function of $T$, the norms of $g$ and $g^{-1}$ in $W^{1, \infty}(]-T, T[\times X)$ and the norms of the coefficients of $L$ in $L^{\infty}(]-T, T[\times X)$. This together with (65) entail (62) with $C_{1}=e^{2 T K_{1}}$ for solutions in $\mathcal{F}$ of equation (61) with a source $f \in L_{\text {loc }}^{1}\left(\mathbb{R}_{t} ; L^{2}(X)\right)$.

2. Proof of (63) . The fact that we are dealing with a solution that is locally $H^{2}$ allows us to use the same type of integrations by parts as Lars Hörmander. For $v \in H_{\mathrm{loc}}^{2}(\tilde{X})$ solution of (61), we write

$$
\begin{aligned}
0= & 2 \partial_{t} v\left(\partial_{t}^{2} v-g^{\alpha \beta} \partial_{\alpha \beta}^{2} v+L v-f\right) \\
= & \partial_{t}\left[\left(\partial_{t} v\right)^{2}+g^{\alpha \beta} \partial_{\alpha} v \partial_{\beta} v+v^{2}\right]-2 \gamma^{-1} \partial_{\alpha}\left[\gamma g^{\alpha \beta} \partial_{t} v \partial_{\beta} v\right] \\
& +2 \partial_{t} v L v+2 \gamma^{-1} \partial_{\alpha}\left(\gamma g^{\alpha \beta}\right) \partial_{t} v \partial_{\beta} v-\left(\partial_{t} g^{\alpha \beta}\right) \partial_{\alpha} v \partial_{\beta} v-2 v \partial_{t} v-2 f \partial_{t} v
\end{aligned}
$$

Integrating (66) on the domain $\Omega_{T}^{-}=\{-T \leq t \leq \varphi(x)\}$ for the measure $\mathrm{d} t \mathrm{~d} \nu=\gamma \mathrm{d} t \mathrm{~d} x$, we obtain

$$
\begin{aligned}
0= & \int_{\Sigma}\left(\left(\partial_{t} v\right)^{2}+g^{\alpha \beta} \partial_{\alpha} v \partial_{\beta} v+v^{2}\right) \mathrm{d} \nu_{\Sigma}-\int_{X_{-T}}\left(\left(\partial_{t} v\right)^{2}+g^{\alpha \beta} \partial_{\alpha} v \partial_{\beta} v+v^{2}\right) \mathrm{d} \nu \\
& +\int_{\Sigma} 2 g^{\alpha \beta} \partial_{t} v \partial_{\alpha} \varphi \partial_{\beta} v \mathrm{~d} \nu_{\Sigma} \\
& +\int_{\Omega_{T}^{-}}\left(2 \partial_{t} v L v+2 \gamma^{-1} \partial_{\alpha}\left(\gamma g^{\alpha \beta}\right) \partial_{t} v \partial_{\beta} v\right. \\
& \left.-\left(\partial_{t} g^{\alpha \beta}\right) \partial_{\alpha} v \partial_{\beta} v-2 v \partial_{t} v-2 f \partial_{t} v\right) \mathrm{d} t \mathrm{~d} \nu
\end{aligned}
$$

The first three terms give (using the fact that $\Sigma$ is totally null)

$$
\begin{gathered}
-E(-T, v)+\int_{\Sigma}\left(\partial_{t} v\right)^{2} \mathrm{~d} \nu_{\Sigma}^{0}+\int_{\Sigma}\left(g^{\alpha \beta}\left(\partial_{\alpha} v+\partial_{\alpha} \varphi \partial_{t} v\right)\left(\partial_{\beta} v+\partial_{\beta} \varphi \partial_{t} v\right)+v^{2}\right) \mathrm{d} \nu_{\Sigma} \\
=-E(-T, v)+\left\|v_{\left.\right|_{\Sigma}}\right\|_{H^{1}(\Sigma)}^{2}
\end{gathered}
$$

and the other terms, thanks to the assumptions on $g$ and the coefficients of $L$, can be estimated by

$$
\begin{aligned}
& C(T, g, L) \int_{-T}^{T} E(t, v) \mathrm{d} t+2 \int_{\Omega_{T}^{-}}\left|\partial_{t} v f\right| \mathrm{d} t \mathrm{~d} \nu \\
& \leq C(T, g, L) \int_{-T}^{T} E(t, v) \mathrm{d} t+\left\|\partial_{t} v\right\|_{L^{\infty}(]-T, T\left[; L^{2}(X)\right)}^{2}+\|f\|_{L^{1}(]-T, T\left[; L^{2}(X)\right)}^{2} \\
& \leq C(T, g, L) \int_{-T}^{T} E(t, v) \mathrm{d} t+\sup _{t \in]-T, T[} E(t, v)+\|f\|_{L^{1}(]-T, T\left[; L^{2}(X)\right)}^{2},
\end{aligned}
$$

where $C$ has the required continuity properties. Estimate (62) then gives (63).

3. Proof of (64). For the converse inequality, for $\min \varphi \leq t \leq T$, we integrate (66) on the domain $\Omega_{t}^{+}=\{\varphi(x) \leq s \leq t\}$, i.e. the set of points of $\overline{\tilde{X}}$ situated in the future of $\Sigma$ and in the past of $X_{t}$. Following Hörmander, we put

$$
E_{\varphi}(t, v)=\int_{\varphi(x) \leq t}\left(\left(\partial_{t} v(t, x)\right)^{2}+g^{\alpha \beta}(t, x) \partial_{\alpha} v(t, x) \partial_{\beta} v(t, x)+v(t, x)^{2}\right) \mathrm{d} \nu .
$$


We obtain

$$
\begin{aligned}
0= & -\int_{\Sigma \cap \Omega_{t}^{+}}\left(\left(\partial_{t} v\right)^{2}+g^{\alpha \beta} \partial_{\alpha} v \partial_{\beta} v+v^{2}\right) \mathrm{d} \nu_{\Sigma}+E_{\varphi}(t, v) \\
& -\int_{\Sigma \cap \Omega_{t}^{+}} 2 g^{\alpha \beta} \partial_{t} v \partial_{\alpha} \varphi \partial_{\beta} v \mathrm{~d} \nu_{\Sigma} \\
& +\int_{\Omega_{t}^{+}}\left(2 \partial_{t} v L v+2 \gamma^{-1} \partial_{\alpha}\left(\gamma g^{\alpha \beta}\right) \partial_{t} v \partial_{\beta} v\right. \\
& \left.-\left(\partial_{t} g^{\alpha \beta}\right) \partial_{\alpha} v \partial_{\beta} v-2 v \partial_{t} v-2 f \partial_{t} v\right) \mathrm{d} t \mathrm{~d} \nu
\end{aligned}
$$

The first three terms give

$$
E_{\varphi}(t, v)-\left\|v_{\left.\right|_{\Sigma}}\right\|_{H^{1}\left(\Sigma \cap \Omega_{t}^{+}\right)}^{2}
$$

and the remainder can be estimated by

$$
C(T, g, L) \int_{-T}^{t} E_{\varphi}(s, v) \mathrm{d} s+\int_{\Omega_{T}^{+}}\left|f^{2}\right| \mathrm{d} s \mathrm{~d} \nu,
$$

where $C$ has the appropriate continuous dependence on $T, g, g^{-1}$ and $L$. Gronwall's inequality entails for $\max \varphi<t \leq T$

$$
E(t, v) \leq \tilde{C}(T, g, L)\left(\left\|v_{\mid \Sigma}\right\|_{H^{1}(\Sigma)}^{2}+\|f\|_{L^{2}\left(\Omega_{T}\right)}^{2}\right),
$$

where $\tilde{C}$ again has the required continuity properties. Eventually, estimate (62) gives (64).

This concludes the proof of proposition 4.2 .

Let us now consider, for $k \in \mathbb{N}$, the solution $u_{k}$ of (53) associated with the initial data $u_{0}^{k}, u_{1}^{k}$ defined above. Using standard energy estimates of type (16), we see that for all $T>0,\left\|u_{k}\right\|_{\mathcal{F}, T}$ is bounded uniformly in $k$. Now consider the equation satisfied by $u_{k}-u_{l}$, for $k, l \in \mathbb{N}$ :

$$
\partial_{t}^{2}\left(u_{k}-u_{l}\right)-g^{\alpha \beta} \partial_{\alpha} \partial_{\beta}\left(u_{k}-u_{l}\right)+\tilde{L}_{1}^{k}\left(u_{k}-u_{l}\right)=\left(\tilde{L}_{1}^{l}-\tilde{L}_{1}^{k}\right) u_{l} .
$$

Let $T>0$, estimate (62) and the hypotheses on $\tilde{L}_{1}^{k}$ give the existence of a constant $C>0$, independent of $k, l$ and of $t \in[-T, T]$ such that, for all $t \in[-T, T]$,

$$
E\left(t, u_{k}-u_{l}\right) \leq C\left(\left\|u_{0}^{k}-u_{0}^{l}\right\|_{H^{1}(X)}^{2}+\left\|u_{1}^{k}-u_{1}^{l}\right\|_{L^{2}(X)}^{2}+\left\|\left(\tilde{L}_{1}^{l}-\tilde{L}_{1}^{k}\right) u_{l}\right\|_{L^{1}(]-T, T\left[; L^{2}(X)\right)}^{2}\right) .
$$

Since

$$
\left(\tilde{L}_{1}^{l}-\tilde{L}_{1}^{k}\right) u_{l}=\left(p_{0}^{k}-p_{0}^{l}\right) \partial_{t} u_{l}+\left(p_{k}^{\alpha}-p_{l}^{\alpha}\right) \partial_{\alpha} u_{l}+\left(q_{k}-q_{l}\right) u_{l},
$$

using (55), the boundedness of $\left\{u_{l}\right\}_{l}$ in $\mathcal{C}^{0}\left([-T, T] ; H^{1}(X)\right) \cap \mathcal{C}^{1}\left([-T, T] ; L^{2}(X)\right)$, a Sobolev embedding $H^{1}(X) \hookrightarrow L^{p_{1}}(X)$ with $p_{1}>2$ and (56) for $p=2 p_{1} /\left(p_{1}-2\right)$ we see that $\left\{u_{k}\right\}_{k}$ converges in $\mathcal{C}\left([-T, T], H^{1}(X)\right) \cap \mathcal{C}^{1}\left([-T, T], L^{2}(X)\right)$. Allowing $k$ to tend to $+\infty$ in equation (53), we see that the limit of $u_{k}$ is the solution $u$ of (4) associated with the data ${ }^{t}\left(u_{0}, u_{1}\right)$ at $t=0$ (this uses the convergence just established as well as (55), (56) and (58)). This convergence and inequality (63) for $u_{k}-u_{l}$ then give that the restriction of $u_{k}$ to $\Sigma$ convergences strongly in $H^{1}(\Sigma)$ (here again we need to use the convergence of $\left(\tilde{L}_{1}^{l}-\tilde{L}_{1}^{k}\right) u_{l}$ towards 0 in $L^{1}(]-T, T\left[, L^{2}(X)\right)$ ). Moreover, using once again the convergence of $u_{k}$ in $\mathcal{C}^{0}\left([-T, T] ; H^{1}(X)\right) \cap \mathcal{C}^{1}\left([-T, T] ; L^{2}(X)\right)$ and standard trace theorems, we see that $u_{\left.k\right|_{\Sigma}}$ converges to $u_{\left.\right|_{\Sigma}}$ in $L^{2}(\Sigma)$. By uniqueness it follows that $u_{\left.k\right|_{\Sigma}}$ converges to $u_{\left.\right|_{\Sigma}}$ in $H^{1}(\Sigma)$. This entails inequalities (20) and (21) for $u$. 
Remark 4.2. It is in this part of the proof that we require a bit more regularity on the metric and the coefficients of the first order terms than in Hörmander's proposed setting, since we need the convergence of $\left(\tilde{L}_{1}^{l}-\tilde{L}_{1}^{k}\right) u_{l}$ towards 0 in $L^{1}(]-T, T\left[, L^{2}(X)\right)$ as $k, l \rightarrow+\infty$. Note that all we need to guarantee this convergence is the convergence of $p_{k}^{0}$ towards $p^{0}$ and of $p_{k}^{\alpha}$ towards $p^{\alpha}$ in $L_{\text {loc }}^{1}\left(\mathbb{R}_{t} ; \mathcal{C}^{0}(X)\right)$ and of $q_{k}$ towards $q$ in $L_{\text {loc }}^{1}\left(\mathbb{R}_{t} ; L^{p}(X)\right)$ for all $p<\infty$. This is true as soon as $g, p^{0}$ and $p^{\alpha}$ belong to $L_{\mathrm{loc}}^{\infty}\left(\mathbb{R}_{t} ; \mathcal{C}^{0}(X)\right)$ and $q \in L_{\mathrm{loc}}^{\infty}(\tilde{X})$. All the rest of the proof is valid for a Lipschitz metric and coefficients of $L$ in $L_{\mathrm{loc}}^{\infty}(\tilde{X})$. Remembering that $p^{0}$ and $p^{\alpha}$ contain first order derivatives of the metric, this remark entails theorem 4.

This shows that under hypothesis (H2), the operator $\mathbb{T}_{\Sigma}$, that to a solution $u$ of (44) in $\mathcal{E}$ associates the trace of the solution $u$ on $\Sigma$ (well defined since the solution is in $\mathcal{F} \hookrightarrow H_{\text {loc }}^{1}(\tilde{X})$ ), is a one-to-one bounded linear operator from $\mathcal{E}$ to $H^{1}(\Sigma)$. It remains to establish the surjectivity.

- $\mathbb{T}_{\Sigma}$ is surjective. Let $v \in H^{1}(\Sigma)$, we prove that there exists $u \in \mathcal{E}$ such that $v=\mathbb{T}_{\Sigma} u$. To do so, we adopt the same regularization procedure as in the second step of the proof of theorem [2] i.e. we consider $\left\{{ }^{k} g\right\}_{k}$ and $\left\{{ }^{k} L_{1}\right\}_{k}$ defined by (a) and (b) and satisfying (35)-(39). We introduce for each $k$ a regularized equation to which we can apply theorem 11 Equation (40) will not do because we cannot guarantee that $\Sigma$ is weakly spacelike for ${ }^{k} g$. In order to make up for this, all we need to do is slow down the propagation speed for (40). We consider a sequence $\left\{{ }^{k} \lambda\right\}_{k},{ }^{k} \lambda \rightarrow 1$ as $k \rightarrow+\infty, 0<{ }^{k} \lambda<1$, such that

$$
\forall k,{ }^{k} \lambda{ }^{k} g^{\alpha \beta}(x, \varphi(x)) \partial_{\alpha} \varphi(x) \partial_{\beta} \varphi(x)<1 \text { almost everywhere on } X \text {. }
$$

We have automatically that $\left\{{ }^{k} \lambda^{-1} k_{g}\right\}_{k}$ satisfies (35), (36) and (39). For each $k$, we define the regularized equation

$$
\frac{\partial^{2} v}{\partial t^{2}}-{ }^{k} \lambda \gamma^{-1} \frac{\partial}{\partial x^{\alpha}}\left(\gamma\left({ }^{k} g^{\alpha \beta}\right) \frac{\partial v}{\partial x^{\beta}}\right)+{ }^{k} L_{1} v=0 .
$$

That is to say, we have slowed down the propagation speed so that $\Sigma$ is now totally spacelike for each equation (68) (i.e. for each $k$ ). We denote by $u_{k}$ the unique solution of (68) in $\mathcal{F}$ such that $\left(u_{k}\right)_{\left.\right|_{\Sigma}}=v,\left(\partial_{t} u_{k}\right)_{\left.\right|_{\Sigma}}=0$ (the existence and uniqueness of such solutions is given by theorem 1). For each $k$, using theorem 11 we have an energy estimate (21) for solutions in $\mathcal{F}$ of equation (68). Using the properties of the regularized metric ${ }^{k} g$ and operator ${ }^{k} L_{1}$, among which the equivalence (uniform in $k$ and locally uniform in $t$ ) between the energy (15) induced by $g$ and that induced by ${ }^{k} \lambda^{-1} k_{g}$, we obtain that $\left\{u_{k}\right\}_{k}$ is bounded in $\mathcal{C}\left([-T, T], H^{1}(X)\right) \cap \mathcal{C}^{1}\left([-T, T], L^{2}(X)\right)$ for any $T>\max \{|\min \varphi|,|\max \varphi|\}$. The rest of the proof follows [9] with elements of the proof of theorem 2 to deal with the regularized metric and operator ${ }^{k} L_{1}$. Extracting a subsequence if necessary, we can conclude that $u_{k}$ converges in the following spaces

$$
H^{1}(]-T, T[\times X)-w, H^{s}(]-T, T[\times X) \text { for all } s<1, L^{\infty}(]-T, T\left[; H^{1}(X)\right)-w-*,
$$

towards a function $u, \partial_{t} u_{k}$ converges towards $\partial_{t} u$ in $L^{\infty}(]-T, T\left[; L^{2}(X)\right)-w-*$. The convergences of ${ }^{k} g,{ }^{k} L_{1}$ and $u_{k}$ allow us to interpret, as in the existence part of the proof of theorem 2] the convergence of each term of equation (68) in a common distribution space, hence $u$ satisfies equation (44) in the sense of distributions. Following again the proof of theorem 2. we show that $u$ belongs to $\tilde{\mathcal{F}}$ and therefore to $\mathcal{F}$. Moreover, the strong convergence in $H^{s}(]-T, T[\times X)$ for all $s<1$ entails the convergence in $L^{2}(\Sigma)$ of the trace of $u_{k}$ on $\Sigma$ towards the trace of $u$ on $\Sigma$. Hence, $u_{\left.\right|_{\Sigma}}=v$. This concludes the proof of theorem 3 .

\section{Acknowledgements}

The author would like to thank Alain Bachelot and Luc Robbiano for helpful discussions while this work was in progress. 


\section{References}

[1] J.C. Baez, I.E. Segal \& Z.F. Zhou, The global Goursat problem and scattering for nonlinear wave equations, J. Funct. Anal. 93 (1990), 239-269.

[2] D. Christodoulou, S. Klainerman, The global nonlinear stability of the Minkowski space, Princeton Mathematical series 41, Princeton University Press 1993.

[3] P. Chrusciel \& E. Delay, Existence of non trivial, asymptotically vacuum, asymptotically simple space-times, Class. Quantum Grav. 19 (2002), L71-L79, erratum Class. Quantum Grav. 19 (2002), 3389.

[4] P. Chrusciel \& E. Delay, On mapping properties of the general relativistic constraints operator in weighted function spaces, with applications, Mémoires de la S.M.F. 94 (2003), 103 pages.

[5] J. Corvino, Scalar curvature deformation and a gluing construction for the Einstein constraint equations, Comm. Math. Phys. 214 (2000), 137-189.

[6] J. Corvino \& R.M. Schoen, On the asymptotics for the vacuum Einstein constraint equations, gr-qc 0301071, to appear in Jour. Diff. Geom.

[7] F.G. Friedlander, Radiation fields and hyperbolic scattering theory, Math. Proc. Camb. Phil. Soc. 88 (1980), 483-515.

[8] F.G. Friedlander, Notes on the wave equation on asymptotically Euclidean manifolds, J. Functional Anal. 184 (2001), 1-18.

[9] L. Hörmander, A remark on the characteristic Cauchy problem, J. Funct. Anal. 93 (1990), $270-277$.

[10] S. Klainerman \& F. Nicolò, Peeling properties of asymptotically flat solutions to the Einstein vacuum equations, Class. Quantum Grav. 20 (2003), 14, 3215-3257.

[11] L.J. Mason \& J.-P. Nicolas, Conformal Scattering and the Goursat problem, J. Hyperbolic. Diff. Eq. 1 (2004) 2, 197-233.

[12] R. Penrose, Null hypersurface initial data for classical fields of arbitrary spin and for general relativity, in Aerospace Research Laboratories report 63-56 (P.G. Bergmann), 1963. Reprinted (1980) in Gen. Rel. Grav. 12, 225-264. 